\section{Astrocytes as regulators}

That astrocytes are more than merely supportive elements of the nervous system is no longer news: in recent years they have been linked to multiple processes, including the regulation of synapse formation and neuronal activity. Filosa et al. now show that neuron-astrocyte interactions that regulate glutamate transport activity can modify hippocampal synaptic plasticity.

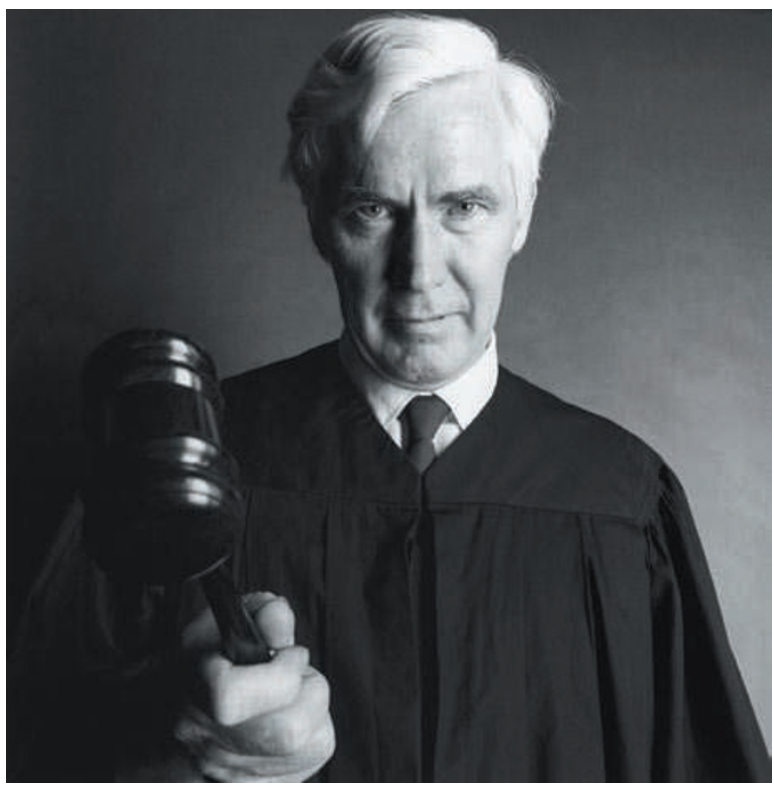

Ephrin type-A receptor 4 (EPHA4) has previously been shown to be necessary for long-term potentiation (LTP) at the synapse between CA3 neurons and CA1 neurons in the hippocampus. Filosa et al. extended this finding, showing that it is EPHA4 expression in the postsynaptic cell (CA1) that is crucial for the induction of LTP by theta-burst stimulation (TBS) in mouse hippocampal slices. Crucially, the loss of ephrin A3, which interacts with EPHA4 and is expressed by astrocytes, also reduced LTP, suggesting that the binding of dendritic EPHA4 to astrocytic ephrin A3 is important for LTP.

Astrocytes can modulate synaptic function through their role in glutamate reuptake at the synapse. The authors showed that the astrocytic glutamate transporters that mediate this process, GLAST (also known as SLC1A3) and GLT1 (also known as SLC1A2), were upregulated in the brains of mice lacking EPHA4 or ephrin A3. By contrast, transgenic overexpression of ephrin A3 reduced glutamate transporter expression.

These findings indicated that EPHA4-ephrin A3 signalling acts to limit glutamate transporter expression and thereby might regulate glutamate reuptake at the synapse. To assess glutamate levels at the synapse the authors measured the degree to which a low-affinity competitive glutamate receptor antagonist was able to inhibit miniature excitatory postsynaptic potentials (with higher levels of inhibition being indicative of lower levels of synaptic glutamate). As predicted, loss of EPHA4 was correlated with lower synaptic glutamate levels.

Further evidence that astrocytic glutamate transport mediates the effects of EPHA4 on LTP was provided by experiments showing that blocking glutamate transport reversed the effects of EPHA4 or ephrin A3 loss.

These findings show that, by regulating the levels of synaptic glutamate, neuron-astrocyte interactions mediated by EPHA4ephrin A3 signalling modulate neuronal plasticity. Whether EPHA4-ephrin A3 signalling is modified by neuronal activity and what downstream pathways regulate transporter expression remain open questions for future research.

Katherine Whalley

ORIGINAL RESEARCH PAPER Filosa, A. et al. Neuron-glia communication via EphA4/ ephrin-A3 modulates LTP through glial glutamate transport. Nature Neurosci. 6 Sep 2009 (doi:10.1038/nn.2394) 